\title{
Intracranial Bony Canal of the Middle Meningeal Artery - Morphological and Histological Analysis
}

\author{
By
Michio FUJIMOTO ${ }^{1,2 *}$, Naruhito OTSUKA ${ }^{1}$, Hiromitsu EZURE ${ }^{1}$, Hiroshi MORIYAMA ${ }^{1}$, Yuriko INOUE ${ }^{1}$, Ryoichi MORI ${ }^{1}$ \\ ${ }^{1}$ Department of Anatomy, Showa University School of Medicine, Shinagawa-ku, Tokyo 142-8555, Japan
${ }^{2}$ Department of Neurosurgery, Shin-yurigaoka General Hospital, Kawasaki, Kanagawa 215-0026, Japan
}

-Received for Publication, December 1, 2016-

\begin{abstract}
Key Words: microsurgical anatomy, meningeal arteries, bony canal, craniotomy
Summary: The middle meningeal artery (MMA) can play an important role in the surgical revascularization. However, the MMA can be easily injured if it passes through a bony canal. We investigated the morphological and histological features of the bony canal to improve surgical results. Materials and Methods: Fifty adult dry skulls were investigated. The length of the bony canal and the distance from the orbital rim to the bony canal were measured. Additionally, 28 cadaveric heads were examined histologically. Results: Sixty-three bony canals were found in 43 skulls. The mean length of bony canals was $9.2 \mathrm{~mm}$, and the mean distance from the orbital rim was $24.0 \mathrm{~mm}$. The bony canal ran mainly from the sphenoid bone $(69.8 \%)$ to the parietal bone (73.0\%). Histologically, both sides of the meningeal grooves gradually closed the distance, and formed the bony canal. The MMA inside the bony canal was enveloped with collagen tissues, divided into branches, and was accompanied by the vein. Conclusions: The bony canal is located around the pterion and is formed during bone growth. The MMA is covered with collagen tissues inside the bony canal. It is possible to safely expose and preserve the MMA during craniotomy with careful drilling.
\end{abstract}

\section{Introduction}

Surgical revascularization for the treatment of chronic ischemic cerebral disease helps to prevent further ischemic stroke. The meningeal arteries can play an important role in the surgical revascularization, particularly in indirect bypass surgery such as encephalo-duro-arterio-synangiosis, induces angiogenesis and produces collateral circulation between the brain surface and the temporal muscle or dura mater. In addition, reversed durapexia, which has been reported as an indirect revascularization method, induces angiogenesis between the brain and reversed dura mater and indicates the significance of the meningeal artery for indirect revascularizations [1]. When performing these indirect methods, it is important to preserve the middle meningeal artery (MMA), which supplies donor tissue, during craniotomy.

After the MMA enters the cranium through the foramen spinosum, the MMA runs anterolaterally and ascends to the pterion along the greater sphenoid wing (temporal segment). Then, the anterior branch of the MMA runs around the pterion (pterional segment) and ascends along the coronal suture. Along this course, a bony canal structure has been reported around the temporal and pterional segment of the MMA in $49-75 \%$ of skulls [2,3]. In cases in which the anterior branches of the MMA are located in a bony canal, the MMA can be injured while removing the bone flap. For preserving the MMA during fronto-temporal craniotomy, the MMA should be exposed by drilling around the pterion [4]. The morphological and histological structure of the middle meningeal groove need to be understood so that the MMA can be safely exposed and preserved. Although several studies have shown that the bony canal varies greatly in terms of length and location, no studies have assessed the microscopic morphology of the inner structure of the bony canal, including the dura mater and the MMA.

With the above in mind, the present study aimed to identify the course of the bony canal and to describe the 
A

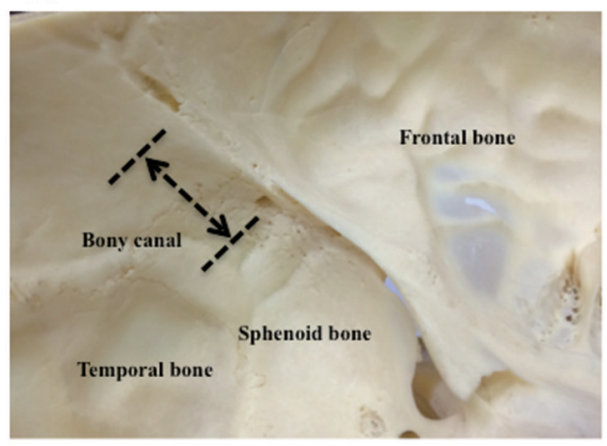

\section{B}

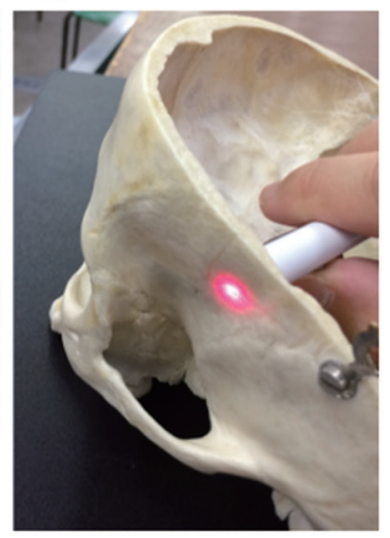

E

D

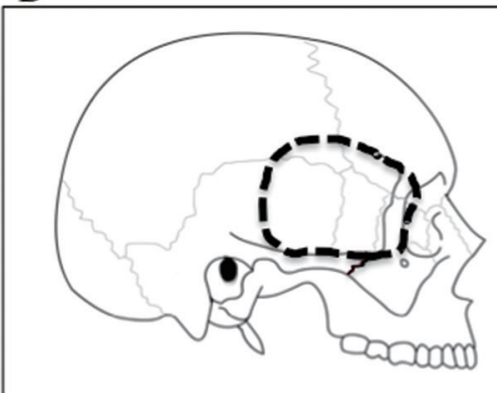

C

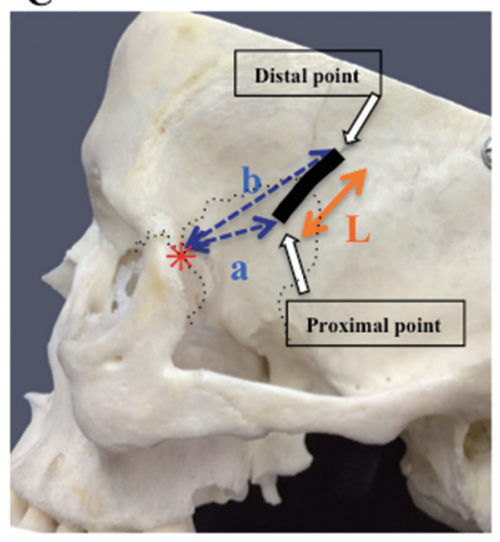

F

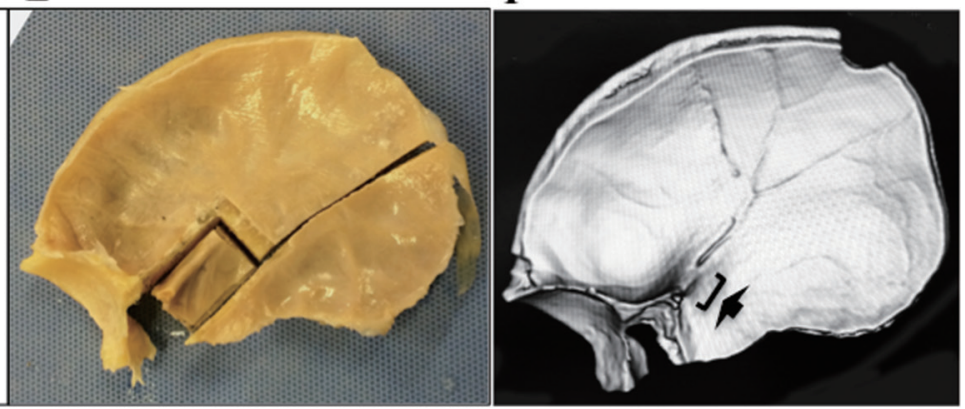

Fig. 1. Inner surface of the dry skull (A). The dotted line indicates the length of the bony canal. The proximal and distal points of the bony canal are illuminated with a laser pointer from the inner surface of the skull (B). Outer surface of the skull (C). Distance from the intersection of the frontozygomatic suture and sphenofrontal suture (landmark; *) to the proximal point of the bony canal (a). Distance from the landmark to the distal point of the bony canal (b). Length of the bony canal (L). The range of extended front-temporal craniotomy (D). A wet cadaver specimen (E). A small piece of specimen including bony canal was cut with an electronic high-speed drill. Three-dimensional computed tomography reveals the bony canal (arrow) and meningeal groove $(\mathrm{F})$.

morphological and histological features of the bony canal structure to allow the MMA to be safely exposed and preserved during neurological surgery.

\section{Materials and Methods}

1) Observations of the dry skull

Fifty adult dry skulls were selected. Cadaver heads were obtained in accordance with the protocol approved by the University. Informed consent was obtained from all patients for inclusion in the study. We investigated observation items (1-5) regarding the bony canal structure, as follows.

\section{(1). The presence of bony canals}

The side on which the bony canal occurred was investigated.

\section{(2). The length of bony canals}

The distance from the proximal and distal points of the bony canal was measured (Fig. 1A).

\section{(3). The course of bony canals}

The proximal and distal points of the bony canals were plotted on the skull to show the course of the bony canal (Fig. 1B).

\section{(4). The length from the lateral orbital rim to bony canal}

The intersection of the fronto-zygomatic suture and spheno-frontal suture was set as a landmark of the lateral orbital rim. The distance from the lateral orbital rim to the bony canal was measured as a guide for indicating the location of the bony canal (Fig. 1C).

2) Histological evaluation of the bony canal

\section{(1). Craniotomy}

Adult cadaveric heads were dissected through extended fronto-temporal craniotomy from the upper part of the superior temporal line, including the lateral orbital rim, to the lower part of the temporal and sphenoid bone. One side of the skull was cut without separating it from 
the dura mater (Fig. 1D, E).

(2) Evaluation of the bony canal, using 3-dimensional computed tomography (3D-CT) (SOMATOM Definition, Siemens Healthcare, Erlangen, Germany)

All of the excised specimens were examined without separating them from the dura by 3D-CT to detect the bony canal structures (Fig. 1F).

\section{(3) Preparation of decalcified sections}

The bone specimens were cut into 1-2 cm blocks including the bony canal and decalcified in $17.7 \%$ EDTA prior to cutting the tissue for histological examination. The obtained specimens were cut in two directions; 1 : vertical relative to the bony canal, 2 : parallel to the bony canal. They were then embedded in paraffin. The specimens were cut into sections of 3-5 $\mu \mathrm{m}$ thickness, using a sliding microtome, and then were stained using hematoxylin and eosin ( $\mathrm{H} \& \mathrm{E})$. Masson's trichrome staining was also used to identify collagen fiber to demonstrate the morphological identity or differences between the dura mater and the tissues in the bony canal. Using a light microscope, serial sections of the bony canal were analyzed to assess the morphological features of the MMA, dura mater, and skull. Adjacent images stitched using image processing software (Adobe Photoshop Elements 13) to provide a wider overview of the bony canal anatomy.

\section{3) Statistical analysis}

The statistical analysis was performed with GraphPad Prism 6 (GraphPad Software Inc., La Jolla, California, USA). Data were analyzed using unpaired t-tests. Correlations were considered significant at $\mathrm{P}<0.05$.

\section{Results}

1) Observation of the dry skull

(1) The presence of bony canals

Among 50 dry skulls, a total of 63 bony canals were found in 43 dry skulls; 15 on the right side, eight on the left, and 20 on both sides. The bony canal was recognized only along the course of the anterior branch of the MMA and never along the course of any other branch of the meningeal artery.

\section{(2) The length of bony canals (Fig. 1C)}

The length of the bony canals was $2-20 \mathrm{~mm}$ (mean: $9.2 \mathrm{~mm}$ ). On right side, it was 1-22 mm (mean: $9.4 \mathrm{~mm}$ ), and on the left side, it was 2-28 mm (mean: $8.9 \mathrm{~mm}$ ). There was no significant difference between the right and left sides $(\mathrm{P}=0.70)$.

\section{(3) The course of bony canals}

a) Inner surface of the skull
The proximal point of the bony canal was located within a limited bone or suture line; 22 cases in the parietal bone $(34.9 \%)$, three in the sphenoid bone $(4.7 \%)$, 24 in the intersection of the spheno-parietal suture and coronal suture $(38 \%)$, nine in the spheno-frontal suture $(14.2 \%)$, four in the coronal suture $(6.3 \%)$, and one in the spheno-parietal suture (1.5\%). The distal point of the bony canal was located in the parietal bone (42 cases, $66.6 \%$ ) and coronal suture (21 cases, $33.3 \%$ ).

\section{b) Outer surface of the skull}

The proximal point of the bony canal was plotted onto the surface of the skull; 44 cases in the sphenoid bone (69.8\%), eight in the temporal bone (12.6\%), seven in the parietal bone $(11.1 \%)$, and four in the sutural bone $(6.3 \%)$. The distal point was plotted onto the parietal bone (46 cases, $73.0 \%$ ), temporal bone (eight cases, $7.9 \%$ ), and sutural bone (four cases, $6.3 \%$ ).

\section{(4) The distance from the lateral orbital rim to the prox-} imal and distal point of the bony canal (Fig. 1C)

The length to the proximal point was $16-32 \mathrm{~mm}$ (mean: $24.0 \mathrm{~mm}$ ) and to the distal point it was $22-51 \mathrm{~mm}$ (mean: $35.6 \mathrm{~mm}$ ).

\section{2) Histological evaluation of the bony canal}

Twenty-eight cadaveric heads (nine female) were examined in this study. The specimens were cut from either side of the head (14 from the right side, 14 from the left side). Only one specimen was excluded because the dura mater was separated from the skull. The bony canal was detected in 12 specimens (six on the right side, six on the left) by 3D-CT. In one case, there was no artery in the meningeal groove predicted by 3D-CT. Six of each specimens were cut vertically or parallel to the bony canal and stained using H\&E and Masson's trichrome.

\section{(1) Serial section vertical to the bony canal}

The MMA was usually covered with the dura mater or collagen tissues on the surface or the inside of the dura mater, and ran accompanied by the meningeal vein (Fig. 2A). The meningeal grooves deepened, and both sides of the meningeal grooves gradually closed the distance and finally formed the bony canal (Fig. 2B-E). The MMA on the dura mater ran into the bony canal, with collagen tissues continuing into the perivascular tissue, and small branch arteries arising from the MMA were recognized in the bony canal (Fig. 2C-E). Furthermore, the anterior branch of the MMA passed through the bony canal along with the MMV. In addition, when the MMA ran on the suture line, the MMA was gradually enveloped bilaterally by the progressive growth of the cranial bones composing the suture line (Fig. 3).

In cases of incomplete bony canals, the roof of the bony canal developed from one side of the vessel (Fig. 4). Thus, the meningeal arteries were partially covered 
A

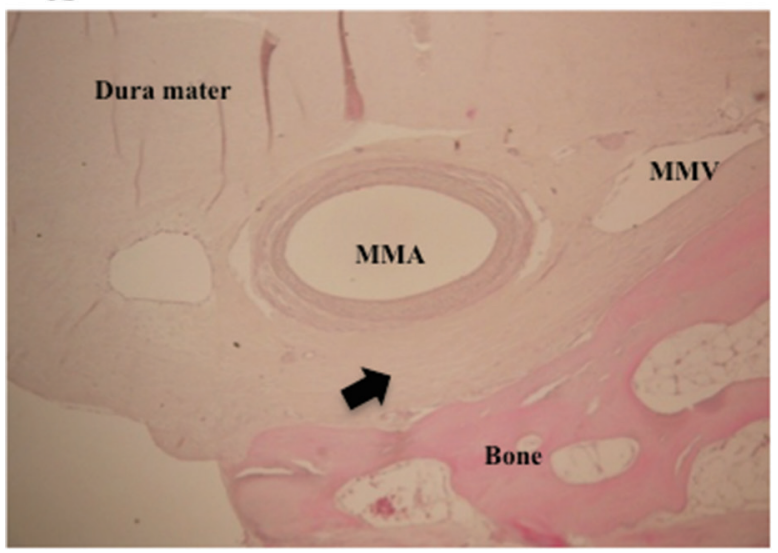

C

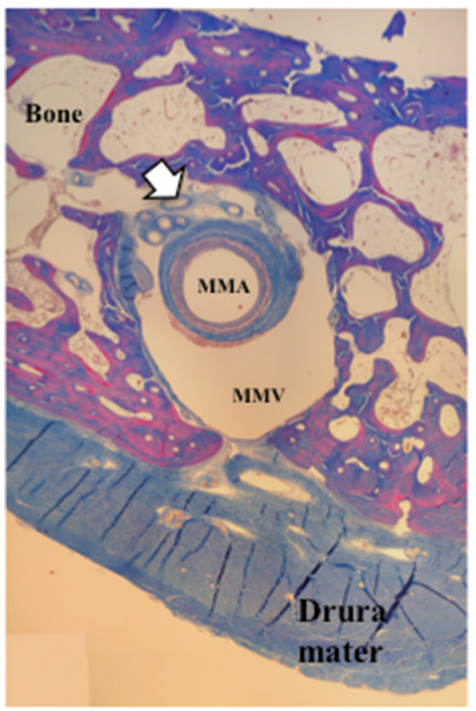

B
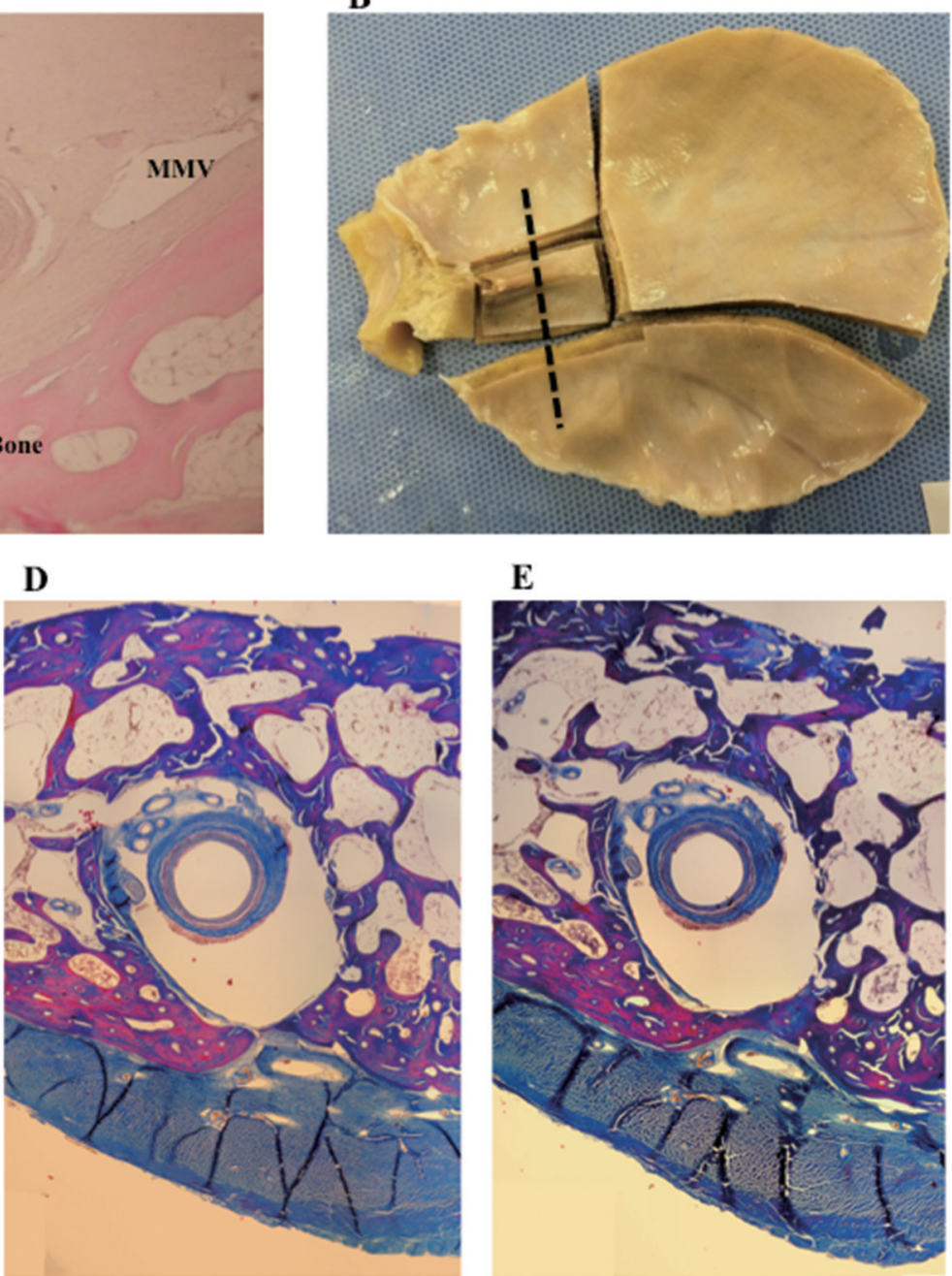

$\mathbf{E}$

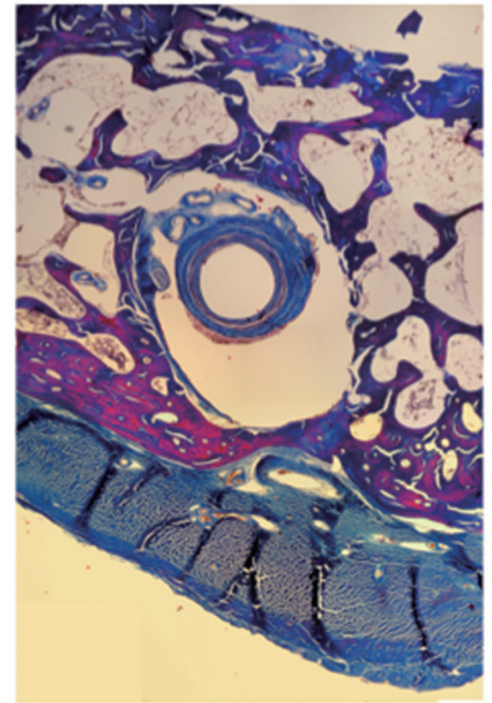

Fig. 2. Vertical section of the anterior branch of the middle meningeal artery (MMA) on the surface of the dura mater (A). The anterior branch of the MMA was covered with dura mater or collagen tissues (arrow). Tissue specimens were cut in the vicinity of the dotted line shown in the wet cadaver specimen (B). Serial section of the specimen vertical to the bony canal (Masson's trichrome stain, $\times 40)(\mathrm{C}-\mathrm{E})$. In the specimens, both sides of the meningeal grooves gradually formed the bony canal. The small arterial branches were accompanied by the middle meningeal artery (white arrows). MMA: middle meningeal artery; MMV: middle meningeal vein.

with the bone and were enveloped with collagen tissue continuing from the dura mater, as in the case of complete bony canals (Fig. 4E).

\section{(2) Serial section parallel to the bony canal}

The MMA passed through the bony canal, with collagen tissue enveloping the MMA at all times (Fig. 5). Furthermore, the collagen tissues were stained with H\&E or Masson's trichrome, like the dura mater, so that the borders between the dura mater and the collagen tissue were unclear (Fig 5C, E, F).

\section{Discussion}

In the present study, we investigated the morphological and histological features of the bony canal through which the MMA passes. The middle meningeal grooves were deepened and gradually enveloped the MMA. The collagen tissues of the outer dural layer continued into the bony canal with the MMA and could be seen in histological studies.

\section{1) Morphological studies}

Several studies have been performed concerning the morphometrics of the skull. According to Plummer's detailed study of the MMA in 1896, 60 bony canals were 


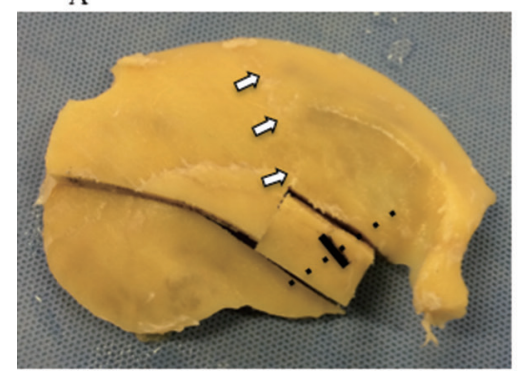

$\mathrm{C}$

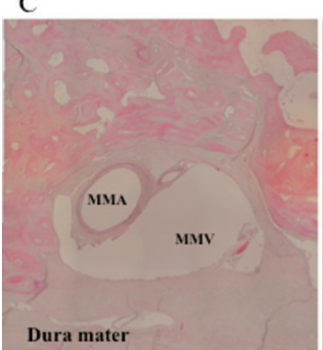

D

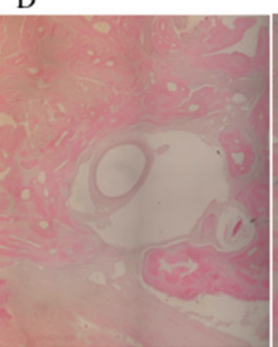

B

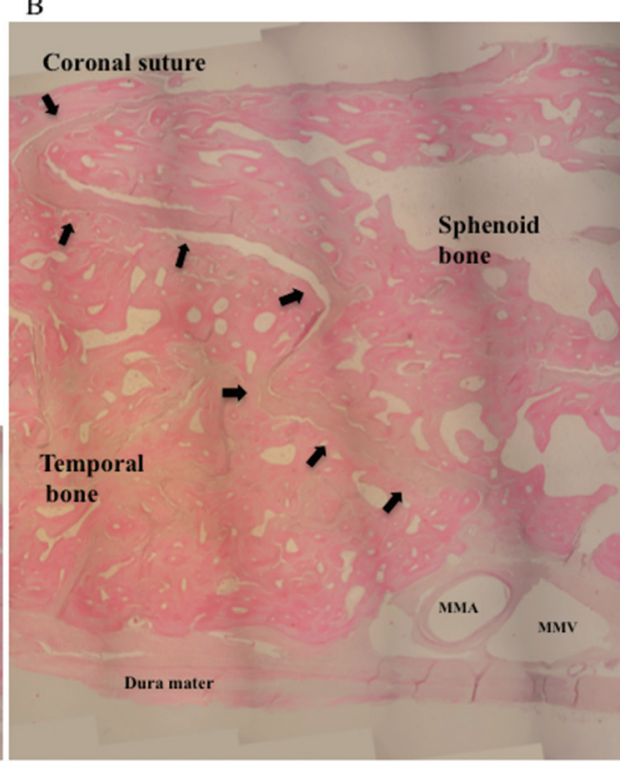

Fig. 3. Serial section of the specimen vertical to the bony canal, which was formed on the suture line (Hematoxylin-Eosin stain, $\times 40)$. Tissue specimens were cut in the vicinity of the dotted line shown in the wet cadaver specimen (A). The white arrows indicate the coronal suture and the solid line indicates the location of the bony canal. The anterior branch of the MMA runs on the suture line (arrows) (B). The meningeal groove was gradually deepened and surrounded by cranial bone (C-E). MMA: middle meningeal artery; MMV: middle meningeal vein.

$\mathbf{A}$

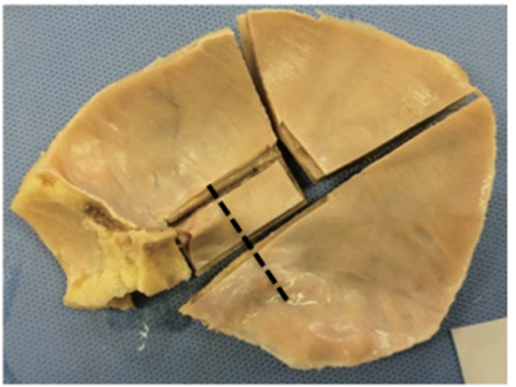

E

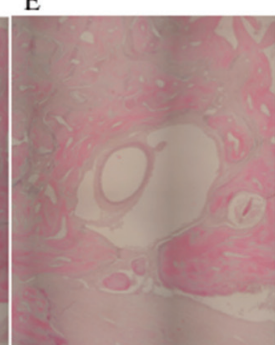


A

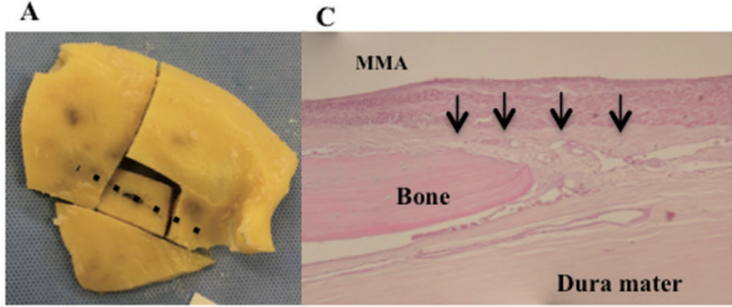

B

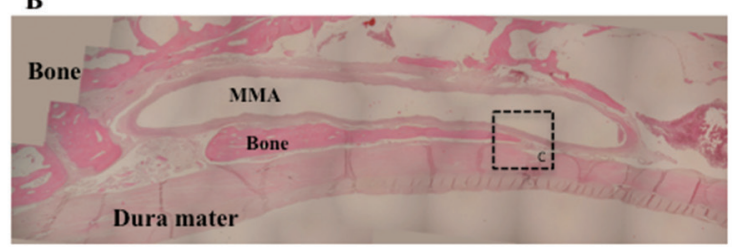

D

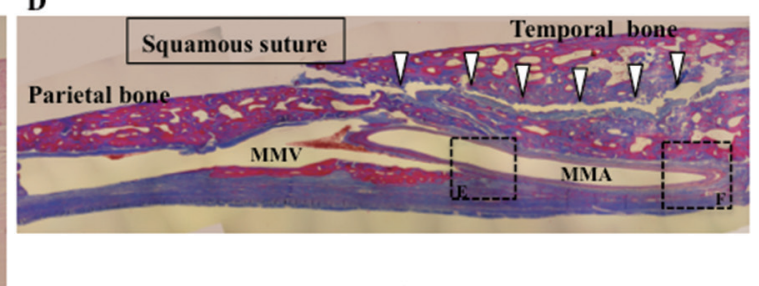

$\mathbf{E}$

F

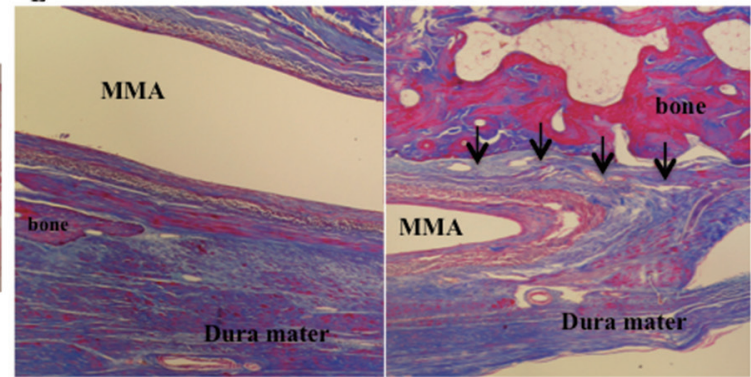

Fig. 5. Serial section of the specimen parallel to the bony canal. Tissue specimens were cut in the vicinity of the dotted line shown in the wet cadaver specimen (A). Two different specimens are shown (B-C; Hematoxylin-Eosin stain, $\times 40$, D-F; Masson's trichrome, $\times 40$,). The middle meningeal artery passed through the bony canal with collagen tissue in the parietal bone. The arrowhead indicates the squamous suture. Higher magnification of the rectangular area in B (C). Higher magnification of the rectangular area in D (E, F). The outer layer of the dura mater, continuing into the bony canal with perivascular tissue (arrows).

seen in 60 cases, and the length of these canals varied from 0.3 to $2.8 \mathrm{~cm}$ [5]. Besides this study, several other authors have reported details of a bony canal structure in the skull $[2,3,6]$. However, previous studies mentioned only the bony canal and not its histological features, including the presence of dura mater and vessels. In this study, the bony canals were recognized in $86 \%$ of the 50 specimens analyzed. In cases in which the MMA ran along the suture line, the MMA was surrounded by cranial bone on both sides, which constituted the suture line. Consequently, the bony canals began and formed on the suture line in $60.4 \%$ of cases. Upon observation from the outer surface of the skull, the bony canal was always located around the pterion, posterior to the coronal suture, inferior to superior temporal line, and never beneath the frontal bone. In addition, the distance from the lateral orbital rim to the proximal point of bony canal was 16-32 $\mathrm{mm}$, and this result indicated that the bony canal never sat within approximately one finger's-width lateral to the lateral orbital rim. Although this location will obviously vary according to the width of the surgeon's finger, it still appears to be a useful landmark in surgical practice.

In terms of the formation of bony canals, the sutural bone was often recognized at the site of the bony canals, and the site was consistent with the sphenoidal fontanelle. In addition, there were some incomplete bony canals covered from just one side. In such cases, the bone on both sides of the MMA gradually closed the distance, and this was confirmed by histological findings. From these results, the bony canals were considered to be formed when the anterior branch of the MMA, which runs along the dura of the sphenoidal fontanelle, was surrounded by cranial bone during fontanelle closure during bone formation.

\section{2) Histological studies}

The anterior branch of the MMA accompanied by the meningeal vein and small arterial branches ran along the surface or the inside of the dura mater. However, the precise inner structure of the bony canal remains unknown. If the MMA is covered with some tissues (including the dura mater) inside the bony canal, these tissues could play an important role in protecting the vessels during skull drilling. Bleeding could easily occur during drilling if the MMA was accompanied by a vein or arterial branches. Therefore, it is very important to understand the morphological and histological inner structures of the bony canal. Histologically, the dura mater has a three-layered architecture: the outermost layer (periosteal layer), meningeal layer, and innermost layer (dural border cell layer) [7, 8, 9]. In addition, a five-layered architecture of the dura mater has been reported based on the orientation of collagen fibers on scanning electron microscopic images [10]. The outermost layer of the dura mater is composed of the inner periosteum of the skull and appears to be attached directly to the skull by extensive collagen fibers [11]. In this histological study, light microscopy observation revealed several interesting findings. The MMA ran along the outermost layer of the dura mater as well as within the meningeal layer, 
and the MMA passing through the bony canal enveloped by collagen tissue, which continued into the outermost layer of the dura mater. These collagen tissues and the outermost layer of the dura mater were considered the same tissue. In addition, the anterior branch of the MMA partially adhered to the surrounding bone because the MMA ran parallel to the MMV and divided into smaller branches inside the bony canal (Fig. 3, 4, 5). Consequently, separating the anterior branch of the MMA from the surrounding bone is considered possible by carefully drilling around the bony canal.

In the present study, we obtained findings that should be useful for preserving the MMA during craniotomy. However, several limitations of the present study should also be acknowledged. First, the age and race of the 50 cases of dry skulls assessed were unknown. Second, our sample size for tissue specimens was smaller than the number of dry skulls included.

\section{Conclusion}

The morphological and histological findings of bony canal formation were investigated. The bony canal was recognized in $86 \%$ of the specimens, and was always located around the pterion. Inside the bony canal, collagen tissue continuing from the outermost layer of the dura mater enveloped the MMA, and the MMA was accompanied by the MMV and small arterial branches. These findings will be useful for surgeons attempting to carefully expose and preserve the anterior branch of the MMA.

\section{Acknowledgements}

We are grateful to those individuals who donated their bodies for research and education on human anatomy without any remuneration. We also thank their families for agreeing to the donation.

\section{Funding}

This research did not receive any specific grant from funding agencies in the public, commercial, or not-forprofit sectors.

\section{Conflict of interest}

none

\section{References}

1) Fujimoto $T$, Asai $J$, Takahashi $M$, Suzuki $R$, Nagashima $G$, Hokaku H, Sato T: Effect of a new treatment, reversed durapexia, for ishchemic lesions. Clin Neurol \& Neurosurg 1997; 99:S62.

2) Harthmann da Silva T, Ellwanger JH, Silva HT, Moraes D, Dotto AC, Viera Vde A, de Campos D: Morphometric analysis of the middle meningeal artery organization in humans - embryological considerations. J Neurol Surg B Skull Base 2013; 74:108-112.

3) Siyan MA, Baillie LJM, Stringer MD: Reappraising the surface anatomy of the pterion and its relationship to the middle meningeal artery. Clin Anat 2012; 25:330-339.

4) Hori S, Kashiwazaki D, Akioka N, Hayashi T, Hori E, Umemura K, Horie Y, Kuroda S: Surgical anatomy and preservation of the middle meningeal artery during bypass surgery for moyamoya disease. Acta Neurochiru 2015; 157:29-36.

5) Plummer SC: Research on the surgical anatomy of the middle meningeal artery. Ann Surg 1896; 23:540-572.

6) Shimizu S, Hagiwara H, Utuki S, Oka H, Nakayama K, Fujii $\mathrm{K}$ : Bony tunnel formation in the middle meningeal groove: An anatomic study for safer pterional craniotomy. Minim Invas Neurosurg 2008; 51:329-332.

7) Nabeshima S, Reese TS, Landis DM, Brightman MW: Junctions in the meninges and marginal glia. J Comp Neurol 1975; 164:127169.

8) Adeeb N, Mortazavi MM, Tubbs RS, Cohen-Gadol AA: The cranial dura mater: a review of its history, embryology, and anatomy. Childs Nerv Syst 2012; 28:827-837.

9) Mack J, Squier W, Eastman JT: Anatomy and development of the meninges: implications for subdural collections and CSF circulation. Pediatr Radiol 2009; 39:200-210.

10) Protasoni M, Sangiorgi S, Cividini A, Culuvaris GT, Tomei G, Dell'Orbo C, Raspanti M, Balbi S, Reguzzoni M: The collagenic architecture of human dura mater. J Neurosurg 2011; 114:17231730.

11) Vandenabeele F, Creemers J, Lambrichts I: Ultrastructure of the human spinal arachnoid mater and dura mater. J Anat 1996; 189:417-430. 